\title{
Evaluating the Effect of Centralization on Routing Convergence on a Hybrid BGP-SDN Emulation Framework
}

\author{
Adrian Gämperli \\ ETH Zurich \\ Zurich, Switzerland \\ gaadrian@student.ethz.ch \\ Vasileios Kotronis \\ ETH Zurich \\ Zurich. Switzerland \\ vkotroni@tik.ee.ethz.ch
}

\author{
Xenofontas \\ Dimitropoulos \\ FORTH \\ Heraklion, Greece \\ fontas@ics.forth.gr
}

\section{MOTIVATION}

A lot of applications depend on reliable and stable Internet connectivity. These characteristics are crucial for missioncritical services such as telemedical applications. An important factor that can affect connection availability is the convergence time of BGP, the de-facto inter-domain routing (IDR) protocol in the Internet. After a routing change, it may take several minutes until the network converges and BGP routing becomes stable again [13]. Kotronis et al. [8,9] propose a novel Internet routing approach based on SDN principles that combines several Autonomous Systems (AS) into groups, called clusters, and introduces a logically centralized routing decision process for the cluster participants. One of the goals of this concept is to stabilize the IDR system and bring down its convergence time. However, testing whether such approaches can improve on BGP problems requires hybrid SDN and BGP experimentation tools that can emulate multiple ASes. Presently, there is a lack of an easy to use public tool for this purpose. This work fills this gap by building a suitable emulation framework and evaluating the effect that a proof-of-concept IDR controller has on IDR convergence time.

\section{Categories and Subject Descriptors}

C.2.2 [Network Protocols]: Routing Protocols ; C.2.3 [Network Operations]: Network Management

\section{Keywords}

BGP; Software Defined Networks; Emulation

\section{OBJECTIVES}

Our primary objective is to support hybrid BGP-SDN experiments with multiple ASes using real router software. This is needed since when deploying a new IDR approach one cannot change the whole infrastructure at once. The framework should take care of configuration management such as IP prefixes and BGP policy templates and the user

Permission to make digital or hard copies of part or all of this work for personal or classroom use is granted without fee provided that copies are not made or distributed for profit or commercial advantage, and that copies bear this notice and the full citation on the first page. Copyrights for thirdparty components of this work must be honored. For all other uses, contact the owner/author(s). Copyright is held by the author/owner(s). SIGCOMM'14, August 17-22, 2014, Chicago, IL, USA.

ACM 978-1-4503-2836-4/14/08

http://dx.doi.org/10.1145/2619239.2631458. should be able to actively control the experiments, e.g., dynamically changing the topology and verifying the effects of changes. Furthermore, it should be possible to easily create topologies based on measured Internet data or theoretical models. This way an experimenter should be able to concentrate more on the experiments and her concept rather than bothering with configuration and management.

Our second objective is to demonstrate the effect of centralization on IDR convergence time. We designed and implemented a proof-of-concept IDR SDN controller that exploits centralization to improve IDR convergence time based on the following design goals. First, the controller should inter-operate with legacy BGP routers. Moreover, the cluster network is transparent to the legacy BGP world, therefore ASes within the cluster maintain their AS identity. In addition, we want to support disjoint AS sub-clusters controlled by the same controller, so that an intra-cluster link failure does not isolate the controlled ASes: paths over the legacy Internet could still connect the sub-clusters.

\section{HYBRID SDN \& BGP EMULATION FRAMEWORK}

The framework is based on a slightly modified version of Mininet [10] that supports the functionality of Quagga [3] - a popular BGP software. The topologies can be either artificial or built from the iPlane Inter-PoP links [12] and the CAIDA AS Relationship [11] datasets. The framework automatically assigns IP addresses and configures network devices, including customer-to-provider and peer-to-peer relationships. To isolate the effects of inter-domain from intradomain routing every AS is emulated by a single network device. This abstraction is useful for use-cases such as ours.

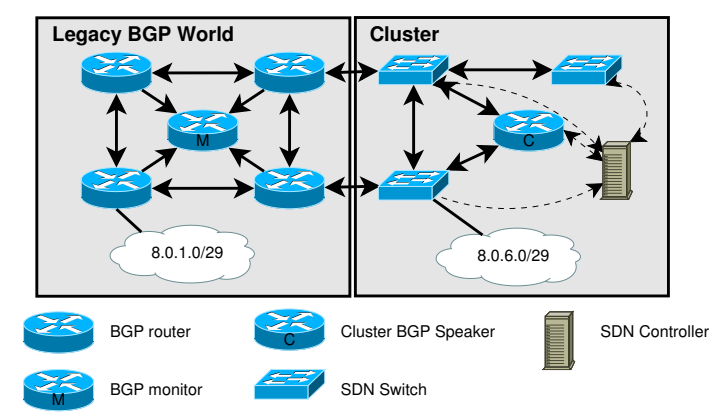

Figure 1: The different components of an example hybrid BGP/SDN emulation experiment. 
In Fig. 1 we show the components of an example experiment. On the left side, we see the legacy BGP part of the emulated network, whereas on the right side we illustrate an SDN cluster, composed of OpenFlow switches. BGP routers and SDN switches can originate prefixes. It is also possible to add hosts with IP addresses within a particular prefix for monitoring end-to-end connectivity with tools like ping, etc. All BGP routers peer with a BGP route collector, which collects routing updates for monitoring purposes. Moreover, within the SDN cluster we have a special BGP speaker, called cluster $B G P$ speaker, which relays routing information between external BGP routers and the SDN controller. The cluster BGP speaker is implemented with ExaBGP [6]. For every BGP peering there is a link from the cluster BGP speaker to the border SDN switch, so as to relay control plane information over the switches.

Experimental setups can be written in Python. We implemented several additional Mininet-BGP commands to announce prefixes, wait until BGP has converged, etc. Additionally, the framework supports tools for automatic log file analysis, network graph creation, convergence time and loss measurement, and route change visualization. For example, to facilitate experiments on IDR stability, the framework detects when the network has converged and whether there is stable connectivity between all hosts. Other compatible tools can be added as Mininet is an extensible platform.

We built an IDR SDN controller over the cluster BGP speaker (using POX [2]), to evaluate the effect of IDR centralization on convergence time. More details on the design and implementation of the IDR controller can be found at [7]. An important insight that we gained is that we can not naively use the same loop avoidance mechanism as BGP, due to the differences between the distributed path selection of BGP and the centralized routing control of SDN. We therefore introduce two graphs for the route selection process: the Switch graph, representing the physical topology of the switches in the cluster and the AS topology graph, which is a transformation of the switch graph per destination prefix. The transformation is restructuring the graph taking carefully into account paths that cross the legacy world and the SDN cluster so as to avoid loops. Best path calculations are based on the Dijkstra algorithm, running on the AS topology graph. AS routes are then compiled to flow rules on the SDN switches. Another design insight we gained is the need for a delayed recomputation of best paths on the controller's side, so as to improve overall stability and rate-limit route flaps due to bursts in external BGP input.

\section{DEMO AND RESULTS}

The demonstration will show the framework and our usecase. One part of the demo will focus on how researchers can use the framework to run and manage experiments [1]. Secondly, we will demonstrate the effect of SDN centralization on IDR convergence time showing visually how it affects an end-to-end video application under different scenarios. Our experiments on a clique topology [7] show that IDR centralization can improve the convergence time even with small SDN cluster deployments. In Fig. 2 we show how the convergence time can be linearly reduced in a route withdrawal experiment with different percentages of SDN deployment in a 16-node clique. On the other hand, route fail-over and announcement experiments did not show this linear improvement, but smaller reductions.

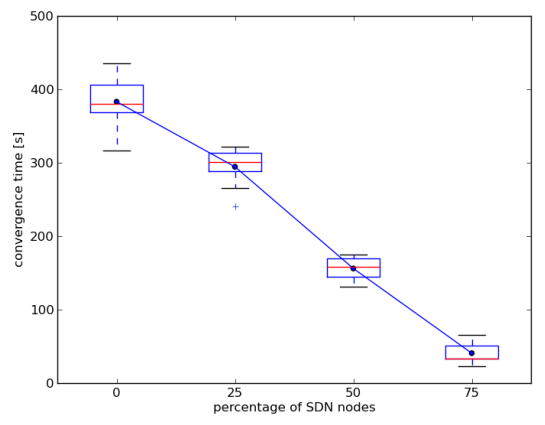

Figure 2: IDR convergence time of route withdrawal on a 16-AS clique topology versus fraction of ASes with centralized route control. The remaining ASes use standard BGP. We show boxplots over 10 runs.

\section{RELATED WORK}

MiniNext [4] is a hybrid SDN - legacy routing emulator based on Mininet and Quagga. However, while MiniNext aims at emulating operational environments and focuses on low-level APIs, our framework focuses on multi-AS IDR experiments and provides a high-level API for experiment lifecycle orchestration. The Open Network Operating System (ONOS) [14] is built to be production ready for large-scale networks. Our approach is better suited for rapid prototyping. Due to simplifications such as cooperative multitasking, we can focus more on research questions than on state consistency and concurrency issues. Finally, RouteFlow [5] is a platform where the controller application mirrors the SDN topology to a virtual network and runs a legacy routing protocol on top of it. Our controller however does not rely on routing decisions of legacy protocols but runs its own algorithms, enabling better integration with SDN concepts.

Acknowledgements: This work was partly funded by European Research Council Grant Agreement n. 338402.

\section{REFERENCES}

[1] http://youtu.be/Cbc8XlIp_C0.

[2] POX. http://www.noxrepo.org/pox/about-pox/.

[3] Quagga Routing Software Suite. http://www .nongnu .org/quagga/.

[4] B. Schlinker et al. Try Before you Buy: SDN Emulation with (Real) Interdomain Routing. In Proc. of ONS, 2014.

[5] C. E. Rothenberg et al. Revisiting Routing Control Platforms with the Eyes and Muscles of Software-defined Networking. In Proc. of ACM HotSDN, 2012.

[6] Exa Networks. Exa-Networks/exabgp. https://github.com/Exa-Networks/exabgp.

[7] A. Gämperli. Evaluating the Effect of SDN Centralization on Internet Routing Convergence. Master's thesis, ETH Zürich, 2014.

[8] V. Kotronis, X. Dimitropoulos, and B. Ager. Outsourcing the Routing Control Logic: Better Internet Routing Based on SDN Principles. In Proc. of ACM HotNets-XI, 2012.

[9] V. Kotronis, X. Dimitropoulos, and B. Ager. Outsourcing Routing using SDN: The Case for a Multi-Domain Routing Operating System. In Poster Proc. of ONS, 2013.

[10] B. Lantz, B. Heller, and N. McKeown. A Network in a Laptop: Rapid Prototyping for Software-defined Networks. In Proc. of ACM HotNets-IX, 2010.

[11] CAIDA. As relationships dataset. http://www. caida.org/data/as-relationships/.

[12] University of Washington. iPlane: Datasets. http://iplane.cs.washington.edu/data/data.html.

[13] Oliveira, Ricardo et al. Quantifying Path Exploration in the Internet. In Proc. of ACM IMC, 2006.

[14] ON.Lab. What is ONOS? http://tools.onlab.us/onos.html. 